\title{
Sleep management protocol to improve screening and management of sleep disorders among HIV population
}

\begin{abstract}
Background: Sleep problems among individuals living with HIV is a major challenge that needs to be addressed.

Objectives: To implement a quality improvement project and develop an evidence based clinic-specific protocol to improve management of sleep disorders among individuals living with HIV served at a local Community Health center.

Methods: Using Pittsburg Sleep Quality Indicator (PSQI) and Insomnia Sleep Index (ISI) questionnaires, individuals living with HIV were assessed for presence of insomnia at baseline and one month after implementation of sleep habit teaching.

Results: A total of 13 individuals living with HIV agreed to participate in the project. The participants in this quality improvement project suffered from sleep disorder as indicated by a mean of 16.40 in the initial ISI and a PSQI score of 9.53 which signified poor sleep quality. A paired-samples t test was calculated to compare the mean initial ISI/PSQI scores to the mean final ISI/PSQI scores. A significant decrease from the initial to final PSQI was found $(\mathrm{t}(12)=3.454, \mathrm{p}=.005)$, indicating improvement to sleep symptoms.

Discussion: One month of clinical interventions among HIV patients indicated that the insomnia was a significant problem as demonstrated by the ISI and PSQI measurements. The results demonstrated unequivocally success. The results are attributable to the patient's motivation to get better, their increased compliance to care, and the staff dedication in educating the patients on sleeping hygiene.
\end{abstract}

Keywords: HIV, individuals living with HIV, insomnia; sleep disorders; health sleep habits

Abbreviations: PSQI, pittsburg sleep quality indicator; ISI, insomnia sleep index; HIV, human immunodeficiency syndrome; SSPS, statistical analysis software; DNP, doctor of nursing practice

\section{Introduction}

Sleep disorders, particularly insomnia, are a major problem among patients with co-occurring HIV and mental illness. Nearly $70 \%$ of individuals suffering from HIV complain of various forms of sleep problems that need to be addressed. ${ }^{1}$ Sleep disturbances can adversely affect patient's well-being and can be debilitating. Poor sleep can lead to impaired cognition, poor concentration, reduced problem solving skills, and impaired judgment. In addition, lack of sleep can cause poor job performance, leading to low productivity, and avoidable accidents at work.

Approximately $74 \%$ of individuals suffering from HIV experience insomnia, with sleep disturbances appearing at all phases of their illness. ${ }^{2}$ This problem is also experienced by individuals living with HIV that receive care in a local community mental health clinic in South Western State. The majority of these patients complaints of insomnia and are on multiple sleep aid medications. Despite the use of sleep medications, the problems of insomnia still persistence requiring alternative methods of managing the problems. Due to this fact, there is a need to find better ways to manage individuals with HIV who are suffering from insomnia. The clinic does not have a standardized protocol for managing insomnia and therefore creates the need to develop an individualized clinic protocol to manage individuals living with HIV that has sleep problems. This quality improvement project
Volume 5 Issue I - 2016

\author{
Serah Muigai,' Kimberly A Williams,' Kisani \\ Ogwaro' \\ 'University of south Alabama, USA \\ ${ }^{2}$ University of South Alabama, College of Nursing, Mobile, \\ Alabama, USA \\ ${ }^{3}$ Department of Psychiatry, University of Arizona, USA
}

Correspondence: Serah Muigai, University of South Alabama, USA, Email smugigai02@gmail.com

Received: July 17, 2015 | Published: January 05, 2016 used an evidenced based standardized sleep-management protocol to manage insomnia more effectively.

\section{Materials and methods}

\section{Background}

Sleep disorders are common among individuals living with HIV. Sleep disturbance is a substantial problem experienced by people living with mental illness and HIV. ${ }^{3-5}$ According to Seda et al., ${ }^{6}$ approximate $73 \%$ of individuals living with HIV often complain of sleep disturbances. More concerning, Gamaldo et al. ${ }^{7}$ found that sleep disturbances and disorders among HIV individuals are some of the major problems among this population, however, these challenges remain unrecognized.Poor sleep quality contributes to poor quality of life, lethargy, depression, and diminished cognitive functioning. ${ }^{6,8,9}$ Babson et al., ${ }^{10}$ found that relationships between patient's medication adherence and individual reports of worsening HIV symptoms were in part affected by sleep quality. Chen et al., ${ }^{11}$ posits that individuals living with HIV should be assessed for presence of any sleep disturbances in every contact with health providers. Identifying the presence of the sleep disorders among this individuals living with HIV was important for early management of insomnia. According to Yang et al., ${ }^{12}$ poor sleep hygiene practices can hamper sleep and can lead to sleep disruptions. Therefore, reduction of maladaptive sleep practices through sleep hygiene education may promote sleep in patients suffering from sleep disorders. According to Homsey \& $\mathrm{O}^{\prime}$ Connell ${ }^{13}$ inadequate and poor sleep caused by insomnia can affect job performance, relationships, and performance of daily routine 
tasks. Early diagnosis and management of insomnia among this population is essential. Clinicians serving individuals living with HIV should therefore assess presence of insomnia at every contact with the patient to promote better management of insomnia and to improve their health and well-being.

\section{Sample}

The sample for the project included men and women living with HIV, who were seen in a local community clinic. The inclusion criteria included patients both male and female adult patients ages 1970, proficient in English, and suffering from HIV. Exclusion criteria included individuals with no HIV, younger than 19 or older than $70 y e a r s$, those not suffering from insomnia, non-English speakers, and those who are unwilling to participate. The initial participants were 16 individuals living with HIV. However, three individuals did not participate in post intervention questionnaires. The three participants were contacted on several occasions without success. Therefore the final sample $(\mathrm{n}=13)$ included seven men and six women.

\section{Procedure}

The project was first approved by the IRB at the Redacted. From February 1, 2015 to March, 30 2015, all patients that visited the clinic were presented with an opportunity to participate in the quality improvement project. Explanation and the purpose of quality improvement project was done, and the informed consent was obtained from the participants in a private room within the clinic. Those individuals who agreed to participate in the project were helped to fill up PSQI and ISI questionnaires, which required about 5-10minutes to complete. After one month the participants will be required to fill new questionnaires with the previous questionnaires.

\section{Measures}

To assess patient acuity of sleep disorder, the DNP utilized two self-report tools. The utilized tools were Pittsburg Sleep Quality Index (PSQI) and Insomnia Severity Index (ISI).Each participant was given a PSQI questionnaire and an ISI questionnaire to fill out. A score of 8 and above on the ISI, and 5 and above on the PSQI indicated the presence of insomnia. The PSQI is a self-rating questionnaire and has complete grade between 0 and 21, encompassing seven sub totals. The questionnaire is easy to utilize by individuals and can be done in about five minutes. The PSQI is used to assess personal sleep quality over the past month. Seven clinical spheres of sleep problems which include; sleep quality, latency, duration of sleep , typical sleep efficacy, disturbances, utilization of medications, and daytime function disturbances are all evaluated by this tool. The PSQI has demonstrated good internal consistency of 0.83 and overall test-retest reliability $0.85 .{ }^{14}$ The ISI questionnaire is a short, tailored survey that calculates insomnia symptoms and their effects. It is comprised of seven items evaluating onset of sleep, sleep preservation, awakening early in the morning, functioning impairment, professed inadequacy endorsed to the lack of sleep, distress about sleep problems, and contentment with individual sleep. ${ }^{15}$ ISI questionnaire is a reliable and valid instrument to identify cases of insomnia in the individual patients, and has demonstrated $86.1 \%$ in sensitivity and $87.7 \%$ specificity. ${ }^{16}$ Therefore, since the ISI questionnaire is easy to utilize, it was an instrument of choice for this quality improvement project.

\section{Intervention}

The quality improvement intervention involved identifying participant's bedtime routine, sleep habits, and other interventions the participants have tried before. In addition, the participants were given education regarding healthy sleep habits and were giving informational pamphlet to take home. The educational intervention consisted of 10 standards of healthy sleep habits and took approximately 5-10minutes. Health sleep habits were discussed with the participants and all questions and concerns were answered. Most commonly reported activities before bed were; watching television, drinking a lot of fluids, and use of computers or phones before going to sleep. Therefore, to help these participants, the ones who drank a lot of fluids were advised to reduce fluid intakes to reduce nocturnal awakening; those who watched Television or used computers or cell phones were advised to turn off the screens 30 minutes to help unwind before bed.

\section{Data analysis}

For the purpose of this project, data obtained was analyzed using statistical analysis software, SPSS, version 21.0. Analysis was considered significant if the value kept is $\mathrm{P}<0.05$ as significant. The use of the paired t-test helped to determine whether there was statistical significance among the individual living with HIV and suffering from the insomnia before and after implementation of quality improvement project.

\section{Results and Discussion}

\section{Results}

A total of 16 individuals living with HIV agreed to participate in the project; however, only 13 participants responded to post intervention questionnaires. The participants in this quality improvement project suffered from sleep disorder as indicated by a mean of 16.40 in the initial ISI. In addition, the participant's mean PSQI score was 9.53 which signified poor sleep quality.

On ISI initial rating, 53.3\% had clinical insomnia, $6.7 \%$ had clinical insomnia-moderate, $13.3 \%$ had clinical insomnia-severe, and 26.7\% had sub threshold insomnia (see Table 1). Patients were given pamphlets and health education on sleeping hygiene for approximately one month, with one session per visit. The patients were compliant in the appointments and received well the health educations. The patients demonstrated understanding by citing techniques of sleep hygiene and stated they had used some of the techniques which helped them to have a better sleep. At the end of the quality improvement project, a post-evaluation on ISI was done to identify the current severity of sleeping problems following health interventions on sleep problems. The rating on ISI at the end of the project yielded the following results: A paired-samples $t$ test was calculated to compare the mean initial ISI/ PSQI scores to the mean final ISI/PSQI scores. The mean on the initial ISI was $13(\mathrm{sd}=3.851)$, and the mean on the final ISI was 11.15 (sd $=4.997)$. A significant decrease from the initial to final ISI was found $(\mathrm{t}(12)=4.674, \mathrm{p}=.001)$, indicating improvement to sleep symptoms. The mean on the initial PSQI was $9.54(\mathrm{sd}=2.259)$, and the mean on the final PSQI was $7.08(\mathrm{sd}=3.252)$. A significant decrease from the initial to final PSQI was found $(\mathrm{t}(12)=3.454, \mathrm{p}=.005)$, indicating improvement to sleep symptoms (Table 2-7 \& Charts 1-3).

\section{Discussion}

One month of clinical interventions among HIV patients indicated that the insomnia is a significant problem as demonstrated by the ISI and PSQI measurements. The ISI at baseline showed that all the project participants had clinical insomnia ranging from sub-threshold to severe insomnia, while the initial PSQI measurement showed that all the patients had poor quality of sleep. Most participants 
complained of having few hours of sleep, waking up multiple times at night, and feeling tired most of the days. Although, many participants believed there was ways they could improve their sleep habits, most of them were unsure of how to go about it. Therefore, providing sleep hygiene education to these individuals was important to them. Clinical intervention which was mainly focusing on patient education regarding healthy sleep habits was done to help the patients have a better quality sleep. Pamphlets containing brief education on healthy sleep habits were given to patients. The pamphlets were discussed with the participants and all the questions were answered. Results from PSQI were individually analyzed to adjust specific teaching tailored to the patient needs.

Table I ISI rating initial

\begin{tabular}{|c|c|c|c|c|}
\hline & Frequency & Percent & Valid Percent & Cumulative Percent \\
\hline Clinical insomnia & 7 & 53.8 & 53.8 & 53.8 \\
\hline Clinical insomnia - moderate & I & 7.7 & 7.7 & 61.5 \\
\hline Valid Clinical insomnia - severe & I & 7.7 & 7.7 & 69.2 \\
\hline Sub threshold insomnia & 4 & 30.8 & 30.8 & 100 \\
\hline Total & 13 & 100 & 100 & \\
\hline
\end{tabular}

Table 2 PSQI rating Initial

\begin{tabular}{lllll}
\hline & Frequency & Percent & Valid Percent & Cumulative Percent \\
\hline Valid Associated with poor & 13 & 100 & 100 & 100
\end{tabular}

Table 3 ISI rating Final

\begin{tabular}{llllll}
\hline & Frequency & Percent & Valid Percent & Cumulative Percent \\
\hline \multirow{4}{*}{ Valid } & Clinical insomnia & 4 & 30.8 & 30.8 & 30.8 \\
& Clinical insomnia moderate & 2 & 15.4 & 15.4 & 46.2 \\
& Noinsomnia & 1 & 7.7 & 7.7 & 53.8 \\
& No clinical insomnia & 4 & 30.8 & 30.8 & 84.6 \\
& Sub thresholdinsomnia & 2 & 15.4 & 15.4 & 100 \\
& Total & 13 & 100 & 100 & \\
\hline
\end{tabular}

Table 4 ISI rating Final

\begin{tabular}{cllll}
\hline & Frequency & Percent & Valid Percent & Cumulative Percent \\
\hline Associated with poor & 8 & 61.5 & 61.5 & 61.5 \\
Valid Good sleep & 5 & 38.5 & 38.5 & 100 \\
Total & 13 & 100 & 100 &
\end{tabular}

Table 5 Paired Samples Statistics

\begin{tabular}{llllll}
\hline & & Mean & N & Std. Deviation & Std. Error Mean \\
\hline \multirow{2}{*}{ Pair I } & ISI Initial & I6 & I3 & 3.85 I & 1.068 \\
& ISI Final & II.I5 & I3 & 4.997 & 1.386 \\
Pair 2 & PSQI Initial & 9.54 & 13 & 2.259 & 0.627 \\
& PSQI Final & 7.08 & 13 & 3.252 & 0.902 \\
\hline
\end{tabular}

Table 6 Paired Samples Statistics

\begin{tabular}{lllll}
\hline & & N & Correlation & Sig. \\
\hline Pair I & ISI Initial \& ISI Final & 13 & 0.671 & 0.012 \\
Pair 2 & PSQI Initial \& PSQI Final & 13 & 0.618 & 0.024 \\
\hline
\end{tabular}

Table 7 Paired Samples Test

\begin{tabular}{|c|c|c|c|c|c|c|c|c|c|}
\hline & & \multicolumn{5}{|c|}{ Paired Differences } & \multirow{3}{*}{$\mathbf{t}$} & \multirow{3}{*}{ df } & \multirow{3}{*}{ Sig. (2-tailed) } \\
\hline & & \multirow{2}{*}{ Mean } & \multirow{2}{*}{$\begin{array}{l}\text { Std. } \\
\text { deviation }\end{array}$} & \multirow{2}{*}{$\begin{array}{l}\text { Std. error } \\
\text { mean }\end{array}$} & \multicolumn{2}{|c|}{$\begin{array}{l}\text { 95\% Confidence interval of the } \\
\text { difference }\end{array}$} & & & \\
\hline & & & & & Lower & Upper & & & \\
\hline Pair I & ISI Initial - ISI Final & 4.846 & 3.738 & 1.037 & 2.587 & 7.105 & 4.674 & 12 & 0.001 \\
\hline Pair 2 & PSQI Initial - PSQI Final & 2.462 & 2.57 & 0.713 & 0.909 & 4.014 & 3.454 & 12 & 0.005 \\
\hline
\end{tabular}

The clinical interventions lasted one month long and were collected and analyzed. The ISI showed that some patients had no symptoms of insomnia, while the PSQI showed that there were patients with HIV that had good sleep. In addition, the healthcare providers were more sensitized to effects of insomnia among this population and were more willing to educate patients on health sleep habits. The results demonstrated that the clinical interventions were unequivocally successful. The results are attributable to the patient motivation to get better, their increased compliance to care, and the staff dedication in educating the patient on sleeping hygiene. Due to the positivity of the project, the pamphlet has been adopted as a sleep education tool in the health facility. 


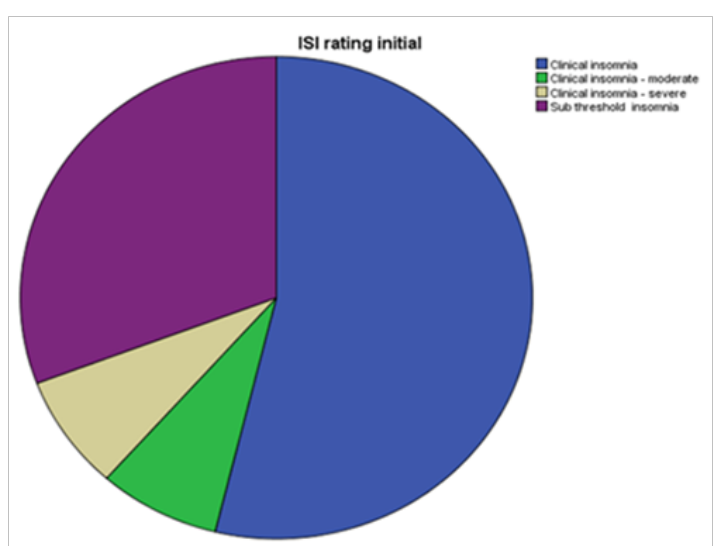

Chart I ISI initial rating.

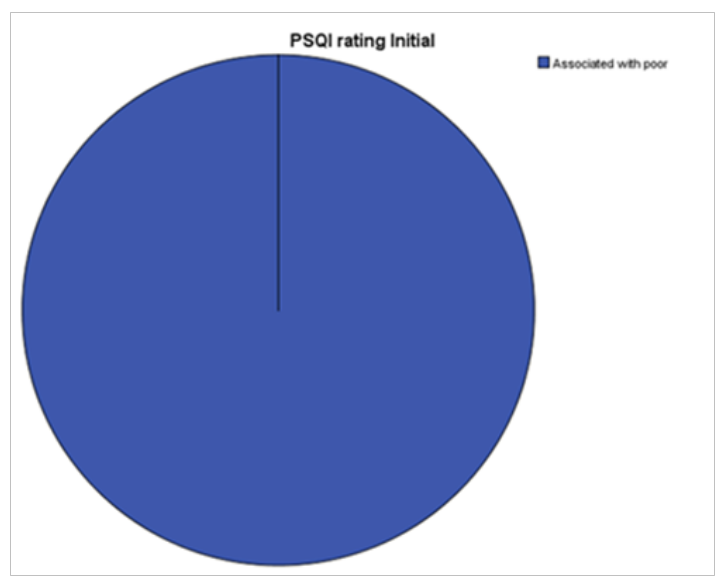

Chart 2 PSQI rating initial.

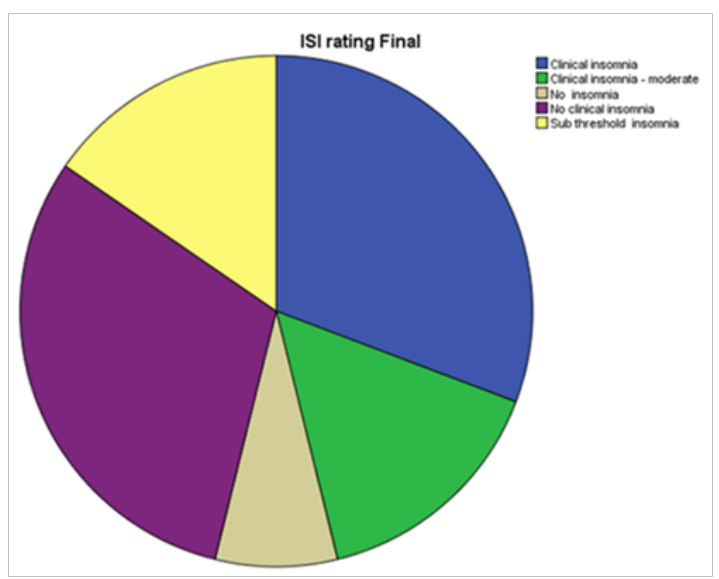

Chart 3 ISI rating final.

\section{Limitations}

Limitations of this quality improvement project included a small sample size, which markedly restricted the power and generalizability. In addition, the participants were obtained from convenience sample carried out in one clinic. Another limitation was that the time allocated for quality improvement project was short, and therefore, little changes were noted on participants and long term changes could not be assessed. Another limitation was that the clinic lost some therapists, and therefore, some patients postponed their appointments or transferred to other agencies in the community. Other patients were not interested with the project as they preferred medications as a quick way of managing their insomnia and were not interested in the sleep hygiene teaching. To overcome this barrier the providers have continued educating and promoting healthy sleep habits as alternative to medications. Another barrier was the EMR. The clinic utilizes electronic Health record which is not user friendly. The EMR software made it difficult to work with a new handout tool. The clinic is currently in a process of implementing a new software. The strength noted was that most participants were motive to participate in the project. In addition, staffs were interested and were eager to see development of a clinic specific sleep hygiene protocol that will ensure patients are well served.

\section{Implications for Nursing Practice}

Practicing nurses working with individuals living with HIV can improve their care by assessing for sleep disorders among this population. Furthermore, providing sleep hygiene education to this population can help promote better sleep; hence, promote patient's health. ${ }^{17}$ Patients reporting presence of sleep problems should be formally assessed using a reliable tool to differentiate the presence of clinical insomnia or of poor quality of sleep.

\section{Conclusion}

Sleep disturbance among individuals living with mental illness and HIV continues to be a major issue affecting this population. Many individuals living with HIV often complain of insomnia, and therefore assessing the presence of sleep problems during every patient contact with the provider is critical. Although, many clinicians provide education regarding health sleep habits, there is a need to have a standardized sleep management protocol to ensure uniformity and to make it easier for clinician, save time, and improve health. A quality improvement project to develop a sleep-management protocol was conducted in a community health clinic to ensure that these individuals are better managed. The ISI and PSQI instruments were utilized during this quality improvement project. The quality improvement project demonstrated that many individuals living with HIV experienced insomnia and were looking for ways to alleviate the problem. The quality improvement project showed that when individuals living with HIV and suffering from insomnia were educated by health providers about the sleep habits, their sleep improved. The quality improvement project helped the clinic to adopt an evidence-based protocol to be used by health care providers at the Community Health Center.

\section{Acknowledgements}

The authors want to appreciate El Rio Community center for allowing this project to conduct at one of their site and thank Brenda McKenna-PMHNP-BC for her support.

\section{Conflicts of interest}

Author declares there are no conflicts of interest.

\section{Funding}

None.

\section{References}

1. Taibi DM. Sleep disturbances in persons living with HIV. J Assoc Nurses AIDS Care. 2013;24(1 Suppl):S72-S85.

2. Webel AR, Moore SM, Hanson JE, et al. Improving sleep hygiene behavior in adults living with HIV/AIDS: a randomized control pilot study of the SystemCHANGETM-HIV intervention. Appl Nurs Res. 2013;26(2):85-91. 
3. Taibi DM, Price C, Voss J. A pilot study of sleep quality and restactivity patterns in persons living with HIV. J Assoc Nurses AIDS Care. 2013;24(5):411-421.

4. Saberi P, Neilands TB, Johnson MO. Quality of Sleep: Associations with Antiretroviral Non-adherence. AIDS Patient Care \& STDS. 2011;25(9):517-524.

5. Jean-Louis G, Weber KM, Aouizerat BE, et al. Insomnia symptoms and HIV infection among participants in the women's interagency HIV study. Sleep. 2012;35(1):131-137.

6. Seda G, Lee-Chiong T, Harrington J. Sleep derangements in centra nervous system infections. Sleep Medicine Clinics. 2012;7(4):703-711.

7. Gamaldo CE, Spira AP, Hock RS, et al. Sleep, function and HIV: A multi-method assessment. AIDS Behav. 2013;17(8):2808-2815.

8. Wirth MD, Jaggers JR, Dudgeon WD, et al. Association of markers of inflammation with sleep and physical activity among people living with HIV or AIDS. AIDS Behav. 2015;19(6):1098-1107.

9. Gamaldo CE, Gamaldo A, Creighton J, et al. Evaluating sleep and cognition in HIV. J Acquir Immune Defic Syndr. 2013;63(5):609-616.

10. Babson KA, Heinz AJ, Bonn-Miller MO. HIV medication adherence and HIV symptom severity: The roles of sleep quality and memory. AIDS Patient Care and STDS. 2013;27(10):544-552.
11. Chen WT, Lee SY, Shiu CS, et al. Fatigue and sleep disturbance in HIVpositive women: A qualitative and biomedical approach. J Clin Nurs. 2013;22(9-10):1262-1269.

12. Yang CM, Lin SC, Hsu SC, et al. Maladaptive sleep hygiene practices in good sleepers and patients with insomnia. J Health Psychol. 2010;15(1):147-155.

13. Homsey M, O'Connell K. Use and success of pharmacologic and nonpharmacologic strategies for sleep problems. J Am Acad Nurse Pract . 2012;24(10):612-623.

14. Insana SP, Hall M, Buysse DJ, et al. Validation of the Pittsburgh Sleep Quality Index Addendum for posttraumatic stress disorder (PSQI-A) in U.S. male military veterans. J Trauma Stress. 2013;26(2):192-200.

15. Yazdi Z, Sadeghniiat-Haghighi K, Zohal MA, et al. Validity and reliability of the Iranian version of the insomnia severity index. Malays J Med Sci. 2012;19(4):31-36.

16. Morin CM, Belleville G, Bélanger L, et al. The insomnia severity index: Psychometric indicators to detect insomnia cases and evaluate treatment response. Sleep. 2011;34(5):601-608.

17. Radtke K, Obermann K, Teymer L. Nursing knowledge of physiological and psychological outcomes related to patient sleep deprivation in the acute care setting. Medsurg Nurs. 2014;23(3):178-184. 